Copyright @ 2007 IEEE. Reprinted from:

L. E. Gurrieri and S. Noghanian, "Multibeam Selection for Indoor MIMO Systems: Two Cases of Study," IEEE Transactions on Antennas and Propagation, vol. 55, no. 4, pp. 1173-1179, 2007.

DOI: 10.1109/TAP.2007.893380, ISSN: 0018-926X

This material is posted here with permission of the IEEE. Such permission of the IEEE does not in any way imply IEEE endorsement of any of the University of Ottawa's products or services. Internal or personal use of this material is permitted. However, permission to reprint/republish this material for advertising or promotional purposes or for creating new collective works for resale or redistribution must be obtained from the IEEE by writing to pubs-permissions@ieee.org.

By choosing to view this document, you agree to all provisions of the copyright laws protecting it. 


\title{
Multibeam Selection for Indoor MIMO Systems: Two Cases of Study
}

\author{
Luis E. Gurrieri and Sima Noghanian, Senior Member, IEEE
}

\begin{abstract}
The performance of multiple-input multiple-output (MIMO) systems using beam selection is investigated in this paper. Based on the results of a channel sounding campaign carried out at the University of Manitoba for line-of-sight (LOS) and non-line-of-sight (NLOS) scenarios, it was possible to contrast the results of transmitter, receiver and joint beam selection in rich scattering environments. The channel was characterized in the 1-2.4 GHz frequency band with a multipath delay resolution better than $5.8 \mathrm{~ns}$. The beam selection was performed by exhaustive search method. The results led us to important conclusions regarding the beam selection procedure and its potential to improve the indoor channel capacity. In LOS, the single input single output (SISO) system that favours the maximum power direction of arrival (DOA) maximizes the capacity. Capacity improvements are observed by increasing the number of receiver beams (RBs) only at high signal-to-noise ratios (SNRs) for omnidirectional transmission. The best performance in transmitter beam selection in LOS is observed by increasing the number of transmitter beams (TBs) for high SNRs. In the case of NLOS, the capacity performance is improved when more than a single beam is used in either, transmitter or receiver side. The joint transmitter-receiver beam selection exhibits best capacity performance only for large SNRs in LOS while the SISO systems outperforms any joint beam selection alternative for low SNRs. In contrast, in NLOS environments, the use of joint beam selection shows a constant capacity performance improvement starting from lower SNR than in the LOS case.
\end{abstract}

Index Terms-Information rate, multiple-input multiple-output (MIMO) systems, multibeam antennas, multipath channels, timedomain measurements.

\section{INTRODUCTION}

$\mathbf{T}$ HE use of spatial multiplexing and diversity by multipleinput multiple-output (MIMO) systems leads to considerable capacity improvements for wireless channels. Diversity methods can be used in different ways to exploit the multiple paths created between transmitters and receivers. In this study, the use of beam selection to increase the capacity of indoor wireless links was investigated.

Multiple-beam selection for MIMO systems exploits the advantages of the rich scattering environments to increment the achievable baud rate. It has been shown [1] that a MIMO system

Manuscript received March 29, 2006; revised November 3, 2006. This work was supported by the University of Manitoba and Natural Sciences and Engineering Research Council of Canada.

L. E. Gurrieri was with the Department of Electrical and Computer Engineering, University of Manitoba, Winnipeg, MB R3T 5V6, Canada. He is now with the Communications Research Centre (CRC) in Ottawa, ON K2H 8S2, Canada (e-mail: lgurrieri@crc.ca).

S. Noghanian is with the Department of Electrical and Computer Engineering, University of Manitoba, Winnipeg, MB R3T 5V6, Canada (e-mail: sima@ee. umanitoba.ca).

Digital Object Identifier 10.1109/TAP.2007.893380 with $N_{T}$ transmitter and $N_{R}$ receiver antennas, can theoretically achieve higher capacity than single antenna systems. In rich scattering environments, it is possible to take advantage of the different spatial signatures of low correlated channels to establish simultaneous links improving the system performance. The only limitation to this idea is the amount of cross-correlation between channels. In most of the practical situations, it is not possible to approximate the theoretical capacity bound of MIMO systems.

In our experiments, instead of using multiple antennas, a virtual multibeam MIMO system was employed to characterize the indoor channel. A multibeam MIMO system uses multiple beams instead of multiple antennas. Virtual multiple-beam system used in measurements activates a single beam at transmitter and a single beam at receiver at any time to measure the channel characteristics. Typical line-of-sight (LOS) and non-line-of-sight (NLOS) scenarios were studied and the performance of these systems was compared. The channel sounding technique made it possible to select the best combination of transmitter-receiver beam angles to maximize the capacity, as a performance estimator. The results show that the incremental beam optimization algorithms proposed by Gharavi-Alkahasari [3] and Gorokhov [4] performs well for this kind of situations.

\section{Measurement SetuP}

\section{A. Floor Plan}

The measurement was carried out in room 528 of the Engineering building of the University of Manitoba. This place was selected because of the large amount of potential scatterers and it represents a typical university office environment. The chosen locations for setups 01 and 02 along with their beam orientations and scanning directions are shown in Fig. 1. The test locations are surrounded by a computer warehouse, multiple offices and other classrooms.

The LOS scenario was represented by setup 01 where the transmitter and receiver antennas were separated by $5.87 \mathrm{~m}$. The transmitting beam (TB) was scanned in $30^{\circ}$ steps counterclockwise where the $0^{\circ}$ reference angle pointed to the left wall as shown in Fig. 1. On the other side, the receiving beam (RB) was scanned counterclockwise at $0^{\circ}, 90^{\circ}, 180^{\circ}$ and $270^{\circ}$. In both cases, the scan was performed in the azimuthal plane [5].

The NLOS Rayleigh-fading channel was tested in setup 02 . The TB was scanned $210^{\circ}, 270^{\circ}$, and $300^{\circ}$ counterclockwise in the same location as setup 01 . The angles were chosen to cover the minimum area of "virtual" LOS between transmitter Tx and receiver Rx. These angles were the most relevant for this study since the use of multibeams to mitigate the correlation between 


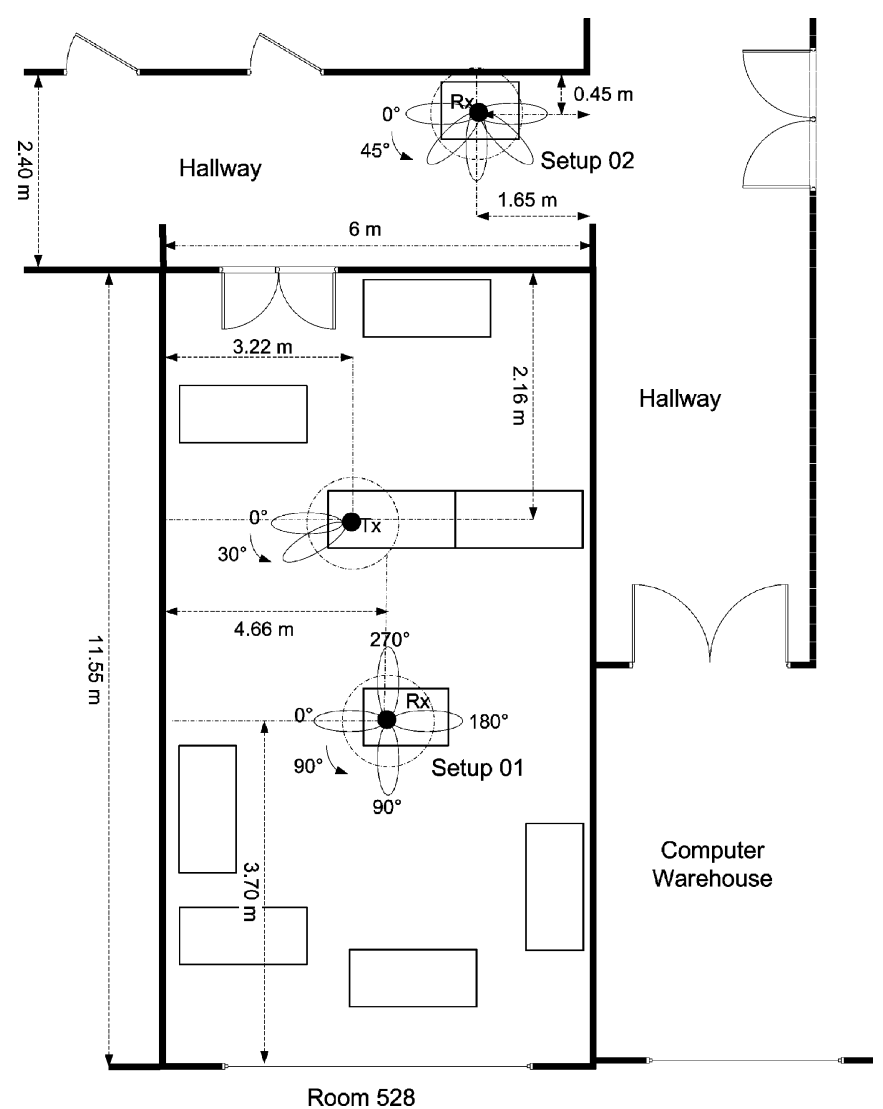

Fig. 1. Test locations for setup 01 and 02.

channels that arise in those directions. The RB in the hallway was scanned at $0^{\circ}, 45^{\circ}, 135^{\circ}$, and $180^{\circ}$ counterclockwise with the reference angle as shown in Fig. 1. The beam angles were selected by taking the physical limitations of setup 02 location into account. The straight-line distance between transmitter and receiver antennas was $4.26 \mathrm{~m}$.

\section{B. Antennas}

Pyramidal horn antennas were used at the transmitter and receiver side. The channel sounding was performed in the $1-2.4 \mathrm{GHz}$ frequency band. This bandwidth (BW) allowed an effective multipath delay resolution of $5.8 \mathrm{~ns}$ after compensating by different window effects. The half-power beam-width (HPBW) of the antennas was $50^{\circ}$ in the E-plane (vertical) and $45^{\circ}$ in the H-plane (horizontal). The scanning angular step used was large enough to detect changes in the multipath pattern.

\section{The Channel Sounding Technique}

A continuous wave (CW) carrier generated by a network analyzer (NA) was used to estimate the complex impulse response (CIR) of the channel. The $6 \mathrm{dBm}$ power $\mathrm{CW}$ was swept from 1.0168 to $2.4 \mathrm{GHz}$ in $2.8 \mathrm{MHz}$ steps. A different CIR estimation was acquired for each combination of TB and RB angles. This technique allowed us to measure the CIR with the delay resolution required to characterize multipath components (MPCs) in indoor environments.
A four terms Blackman-Harris window was applied to reduce the side lobes of the train of pulses in time-domain [10]. The resultant time-domain pulse widening has a lower delay resolution. Equation (1) gives the time-domain pulse width $(\tau)$ as follows:

$$
\tau=\frac{1}{\Delta F} k w
$$

where $\Delta F$ is the frequency sweep range and $k w$ is the widening factor, which is twice the number of window terms. The effective time bin was $5.8 \mathrm{~ns}$ and the pulse train period was $357 \mathrm{~ns}$. Previous results by Saleh-Valenzuela [2] and Spencer et al. [6] for indoor propagation encountered no significant multipath beyond $200 \mathrm{~ns}$ of delay.

Equation (2) was used to calculate the channel impulse response

$$
h_{i, \mathrm{j}}=\sum_{\mathrm{k}=1}^{\mathrm{N}} \sqrt{P_{\mathrm{k}}} \exp \left(j 2 \pi f_{c} \tau_{\mathrm{k}}(t)+\Psi_{\mathrm{k}}(t, \tau)\right) \delta\left(\tau-\tau_{\mathrm{k}}(t)\right)
$$

where $N$ is the number of channel samples, $\Psi_{\mathrm{k}}(t, \tau)$ is the phase of the $k$ th received sample, $i$ and $j$ are the RB and TB angles indices, respectively, and $f_{c}$ is the center frequency in the frequency sweep range. The center frequency in this work was $1.708 \mathrm{GHz}$.

Approximately 10 samples per time bin were considered. Because of applying Blackman-Harris window, the time-binwidth is enlarged by a factor of 10. All the MPC in the original 10 time-binwidths are weighted and added noncoherently.

\section{Signal-to-Noise Ratio}

The signal to noise ratio was estimated from the channel CIR samples. A constant threshold of $30 \mathrm{~dB}$ below the peak signal level was used to distinguish multipaths. It was assumed that the output after the last received multipath was due to noise. The same for the signal detected before the first MPC arrival. Equation (3) was used to estimate the average noise power for $i$ th $\mathrm{RB}$ and $j$ th $\mathrm{TB}$

$$
\overline{\mathrm{N}}^{\mathrm{i}, \mathrm{j}}=\frac{1}{\mathrm{~N}-\mathrm{M}_{\mathrm{l}}-\mathrm{M}_{\mathrm{h}}}\left(\sum_{\mathrm{k}=1}^{\mathrm{M}_{1}-1} \mathrm{P}_{\mathrm{k}}^{\mathrm{i}, \mathrm{j}}+\sum_{\mathrm{k}=\mathrm{M}_{\mathrm{h}}+1}^{\mathrm{N}} \mathrm{P}_{\mathrm{k}}^{\mathrm{i}, \mathrm{j}}\right)
$$

where $M_{l}$ and $M_{h}$ are the number of samples of the first and last paths with strength above the threshold, respectively, $\mathrm{P}_{\mathrm{k}}$ is the power of the $k$ th-sample. The total average power per sample was obtained by considering all the different beam angles and the total number of noise samples.

The average signal-plus-noise (SPN) level at the receiver was estimated using similar considerations, and taking the output samples between $M_{l}$ and $M_{h}$. Equation (4) was used to estimate the average signal power.

$$
\overline{\mathrm{SNP}}^{\mathrm{i}, \mathrm{j}}=\frac{1}{\mathrm{M}_{\mathrm{l}}-\mathrm{M}_{\mathrm{h}}+1}\left(\sum_{\mathrm{k}=\mathrm{M}_{\mathrm{j}}}^{\mathrm{M}_{\mathrm{h}}} \mathrm{P}_{\mathrm{k}}^{\mathrm{i}, \mathrm{j}}\right) .
$$


TABLE I

NOISE, SPN AND SNR ESTIMATED FROM THE MEASUREMENTS

\begin{tabular}{|l|c|c|}
\hline Parameter & Setup 01 & Setup 02 \\
\hline Noise $(\mathrm{dBm})$ & -78.1 & -84.8 \\
\hline SPN $(\mathrm{dBm})$ & -57.7 & -56.9 \\
\hline SNR $(\mathrm{dB})$ & 20.3 & 27.8 \\
\hline
\end{tabular}

Table I contains the estimation results for noise, SPN and signal-to-noise ratio (SNR).

\section{E. Channel Capacity and Beam Diversity}

The capacity of a MIMO system with $N_{T}$ transmitting and $N_{R}$ receiving antennas is given in (5) ([1] and [7])

$$
C=\log _{2}\left[\operatorname{det}\left(I_{N_{R}}+\frac{\mathrm{SNR}}{N_{T}} H H^{H}\right)\right]
$$

where $H$ denotes the channel matrix given by (6), and $H^{H}$ is the conjugate transpose of it

$$
H=\left[\begin{array}{ccc}
h_{11} & \cdots & h_{1 N_{T}} \\
\vdots & \ddots & \vdots \\
h_{N_{R} 1} & \cdots & h_{N_{R} N_{T}}
\end{array}\right]
$$

where $h_{i j}$ is the CIR received by the $i$ th RB due to the transmitted signal originated by the $j$ th TB.

The presence of additive white Gaussian noise (AWGN) was considered. The transmitter had no knowledge of the channel at any given time so the available power is equally divided between the $N_{T}$ TB. Finally, every TB-RB pair was associated to potentially different space channels.

\section{F. The Optimization Method}

The optimization was done by finding the best combination of rows (RBs) and/or columns (TBs) of $H$ that maximizes the system capacity at a given SNR. Initially, the program looks for the row or column with the highest norm and in the following steps, those rows or columns with the maximum orthogonal projection on the subspace that contains the previous selection are chosen. In other worlds, at each step only those rows or columns that lead to the best channel orthogonality are considered. This idea was used before in [3] and [4] for computational efficient, near-optimal search algorithms.

\section{G. Receiver Beam Selection}

The best combination of RBs was found by looking for those rows of the channel matrix that maximize the channel capacity. The assumption of equal power distribution between TBs was held. The optimization results for setup 01 (4 RBs) and setup 02 (5 RBs) are shown in Figs. 2 and 3, respectively.

The case of single-input multiple-output beams (SIMO) is reduced to select the row of the $H$ matrix that maximizes the capacity. The optimization was made assuming that all TB were

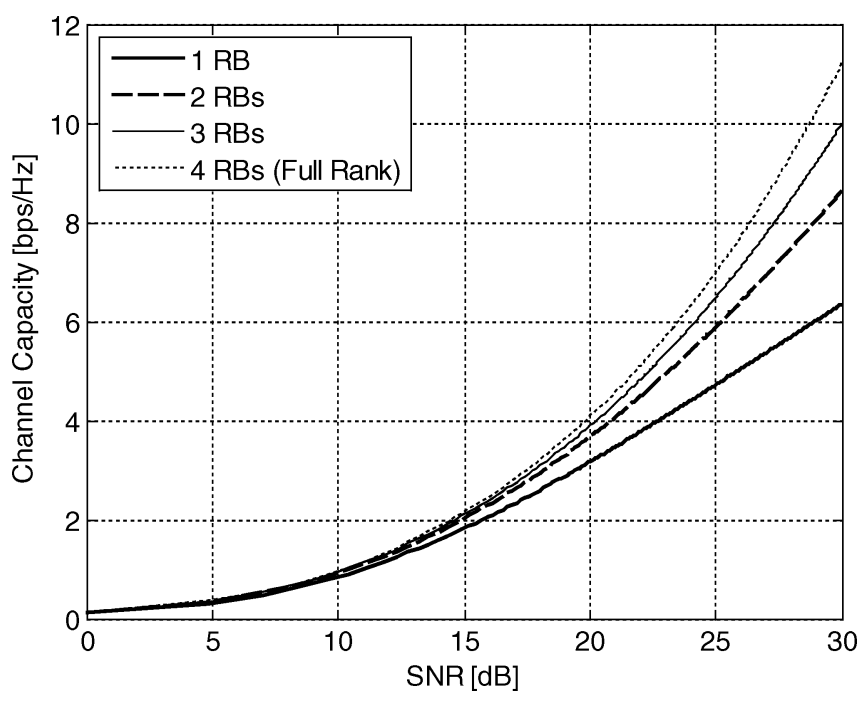

Fig. 2. Receiver beam selection performance for setup 01 (LOS, 12 TBs).

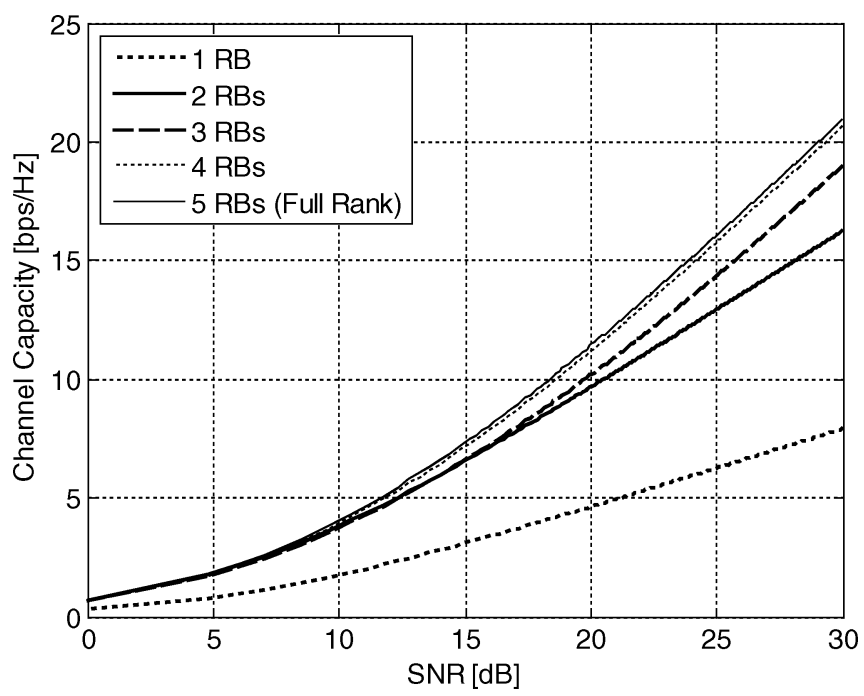

Fig. 3. Receiver beam selection for setup 02 (NLOS, 5 RBs).

used simultaneously. Equation (5) for this particular case can be rewritten as

$$
C_{\mathrm{SIMO}}=\log _{2}\left(1+\frac{\mathrm{SNR}}{N_{T}} \max \left(\operatorname{norm}\left(h_{i}\right)\right)^{2}\right)
$$

where $h_{i}\left(i=1, \ldots, N_{R}\right)$ is a row of the channel matrix $H$. The beam selection problem is reduced to find a vector $h_{i}$ with the maximum squared norm. For setup 1 (NLOS), the optimum SIMO system was associated with the RB at $90^{\circ}$ whereas the optimum solution for setup 2 (NLOS) was linked to the RB at $135^{\circ}$. This result was expected since, for the local scatterers distribution, those beam angles maximize the SNR and consequently the capacity.

It is possible to use the Taylor expansion of the logarithmic expression in (7) in order to maximize the capacity. As it was mentioned, the capacity is proportional to the squared norm of $h_{i}$ only when the higher order terms, which are functions of the SNR, are neglected. This means that, the maximum squared 
TABLE II

Optimum Beam Selection Sequence For Setup 01

\begin{tabular}{|c|c|}
\hline Number of Tx-Beams & Transmitter Beam Number \# \\
\hline 1 & 4 \\
\hline 2 & 46 \\
\hline 4 & 4568 \\
\hline 5 & 45678 \\
\hline 6 & 245678 \\
\hline 10 & 12345678910 \\
\hline
\end{tabular}

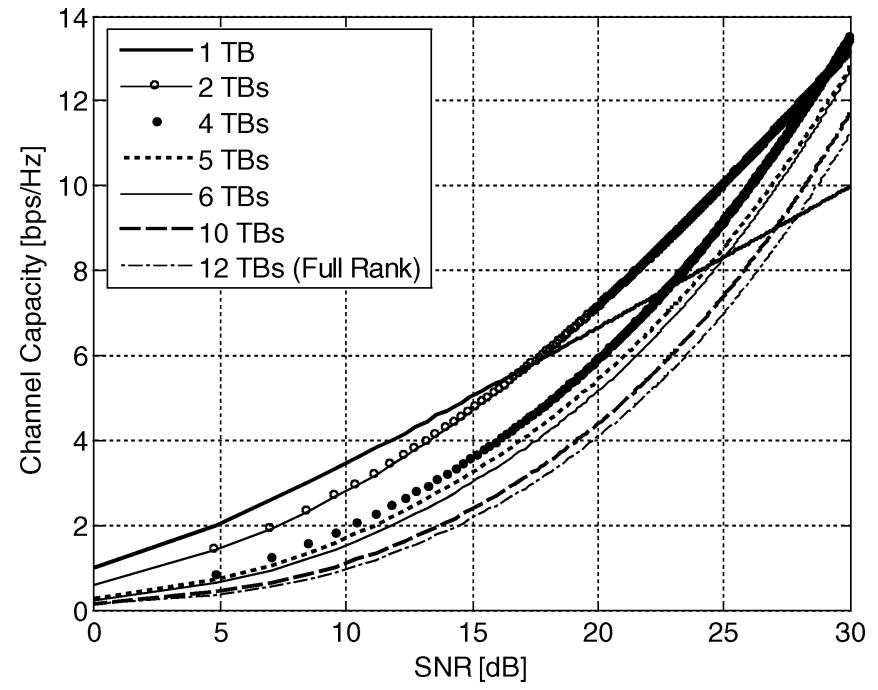

Fig. 4. Transmitter beam selection for setup 01 (LOS, 4 RBs).

norm solution would only be optimal for small SNR levels. For higher SNR, the optimal solution will be more complex than choosing those $h_{i}$ 's with the largest norms. In the next section, this point will be clarified with an example of the optimization results.

For low SNR, it was possible to reduce the system complexity from 4 RBs to 3 or even 2, with a capacity loss of less than $1 \mathrm{bps} / \mathrm{Hz}$ in LOS (Fig. 2). By using 2 RBs instead of 1 , the system improved about $1 \mathrm{bps} / \mathrm{Hz}$ at the operative SNR = $20.3 \mathrm{~dB}$ but further increments in the RB number led to decreasing increments in the capacity gain.

In NLOS (Fig. 3), equivalent performance was achieved by using 4 RBs instead of the full-beam system. For low SNR levels, the number of RBs can be reduced even further attaining equivalent performance as a higher complexity system. In our experiment, for SNR levels below $15 \mathrm{~dB}$, the same capacity was achieved by using 2 RBs instead of 3 RBs. However, for SNR below $10 \mathrm{~dB}$, a system consisting of only $2 \mathrm{RBs}$ performed equivalently well.

\section{H. Transmitter Beam Selection}

The main difference between TB selection and RB selection is that the transmitter should posses some degrees of knowledge

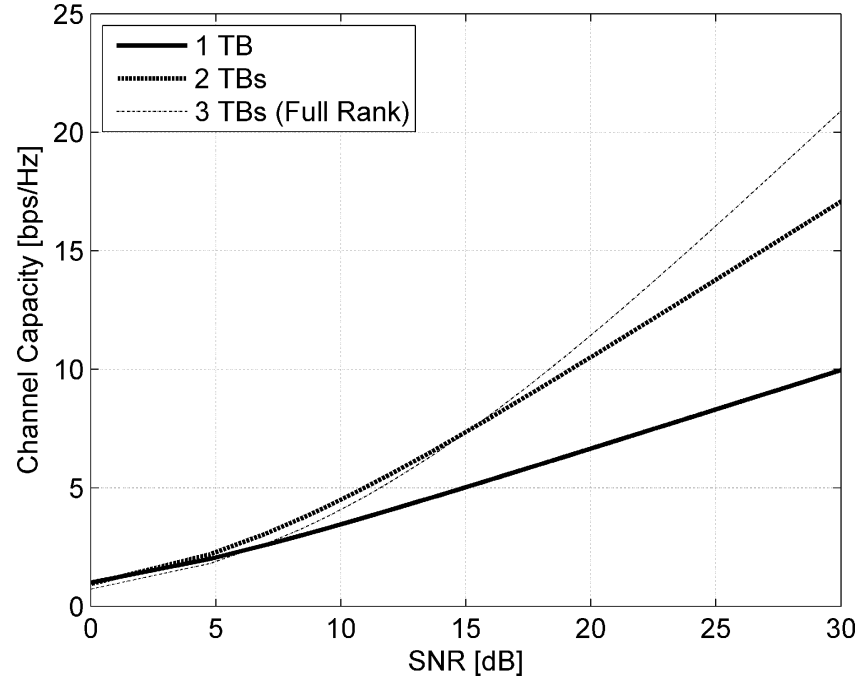

Fig. 5. Transmitter beam selection for setup 02 (NLOS, 5 RBs).

of the channel. When this requirement is fulfilled, the capacity can be optimized by the water-filling technique ([1], [7], and [8]). However, the channel covariance matrix needs to be fed back from the receiver side so the power could be redistributed among the different transmitting antennas.

Alternatively, beam selection can be implemented on the transmitter side to reduce the complexity of a rank deficient channel matrix. By using the TBs that maximizes the channel orthogonality, it is possible to distribute the available power more efficiently. Under the same power constraints, this strategy leads to significant capacity improvement.

Optimization was performed for different TB angles using all the RBs. The results for setup 01 (12 TBs, LOS) and for setup 02 (3 TBs, NLOS) are shown in Figs. 4 and 5, respectively.

The results follow the same idea of selecting the column vectors of the channel matrix (instead of rows for receiver beam selection) with the largest orthogonal projection with respect to subspace that contains the previously selected column vectors (TBs). As an example, let us name the TBs with numbers from 1 to 12 corresponding to angles from $0^{\circ}$ to $330^{\circ}$, respectively, in setup 01. Each of these numbers corresponds to different columns of $H$. The sequence of optimal columns obtained from the exhaustive search optimization algorithm is shown in Table II. 
TABLE III

MAXIMUm NoRm INCREMENTAL BEAm SELECTION FOR SETUP 01

\begin{tabular}{|c|c|}
\hline Number of Tx-Beams & Transmitter Beam Number \# \\
\hline 1 & 4 \\
\hline 2 & 45 \\
\hline 4 & 4568 \\
\hline 5 & 45678 \\
\hline 6 & 345678 \\
\hline 10 & 12345678910 \\
\hline
\end{tabular}

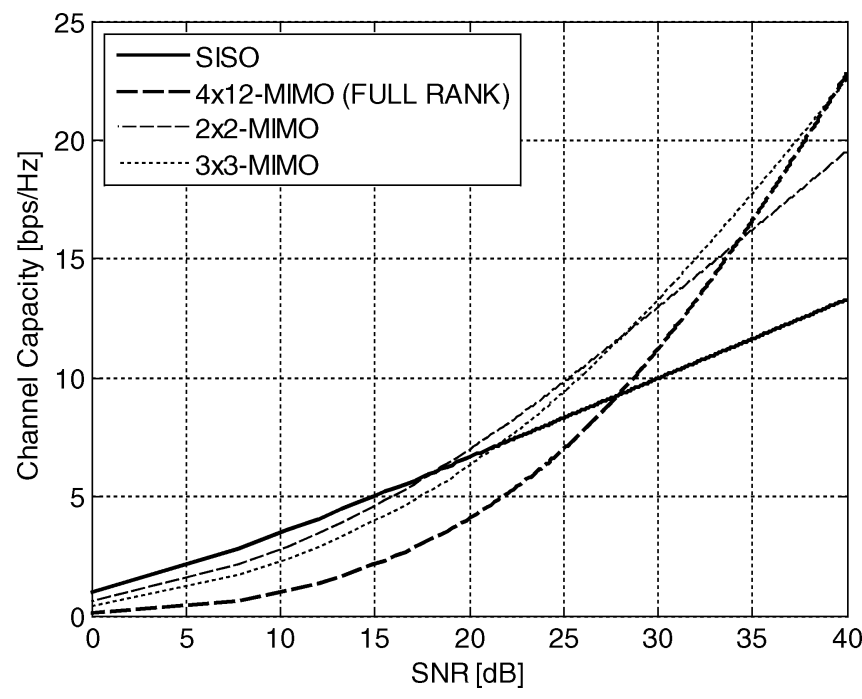

Fig. 6. Joint beam selection for setup 01 (LOS, RBsxTBs-MIMO)

Table III shows the results of using the squared norm that maximized the capacity defined in (7). Both sequences were obtained at the SNR $20.3 \mathrm{~dB}$. TBs were added according to their squared norms in decreasing order. As it was mentioned in the receiver beam selection section, the squared norm method is suitable for low SNR. However, the SNR of $20.3 \mathrm{~dB}$ used to compare the results is large enough to produce erroneous beam sequences after applying the mentioned strategy. Note that the optimal solution shown in Table II suggests choosing beam \#6 for two TB, while the maximum norm method chooses beam \#5 instead. Also for 6 selected beams, the optimal solution uses beam \#2, while the maximum norm uses the beam \#3.

In the LOS case shown in Fig. 4, it is optimum to use a single TB at $90^{\circ}$ for SNR below $17 \mathrm{~dB}$. Notice that decreasing the number of beams implies the redistribution of maximum available power, i.e., for low SNR, instead of distributing the power between $12 \mathrm{TBs}$, all available power could be assigned to a single TB increasing the SNR and therefore increasing the capacity or, alternatively, achieving the same capacity using less transmitting power. However, with the same SNR after the number of TB is reduced, the 2-TB-MIMO surpasses the SIMO system above the $17 \mathrm{~dB}$ SNR threshold and it becomes the optimal solution up to SNR $\sim 28 \mathrm{~dB}$.

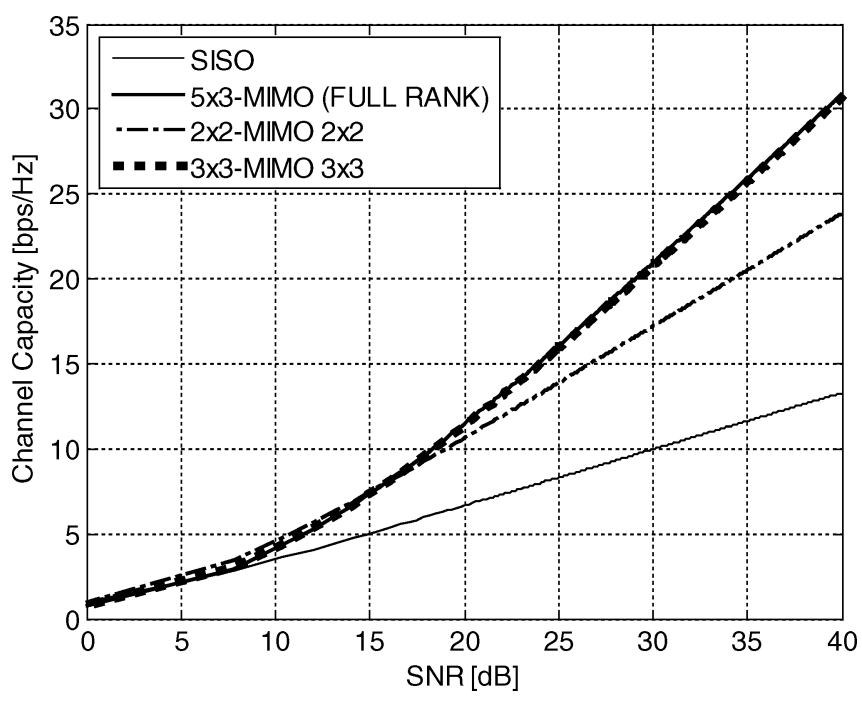

Fig. 7. Joint beam selection for setup 02 (NLOS, RBsxTBs-MIMO).

For the NLOS case, the optimization led to a completely different result, as shown in Fig. 5. For very low SNR $(<1 \mathrm{~dB})$, using $1 \mathrm{~TB}$ is more efficient. For SNR $>15 \mathrm{~dB}$, the capacity increases with the number of TBs.

\section{Joint Beam Selection}

For the joint selection of TB and RB no systematic method exists except for the exhaustive search optimization [8]. Recently, a suboptimal joint selection algorithm was proposed in [9]. A practical solution for this problem would imply an iterative optimization at both receiver and transmitter side. A feedback channel will be required and consequently, the overall complexity of the system will increase. An exhaustive search program was used to find the best joint beam combination. The results for setup 01 and setup 02 are shown in Figs. 6 and 7, respectively.

Interesting results came from the LOS case shown in Fig. 6. For SNR below $18 \mathrm{~dB}$ the SISO system leads to a better performance. Above this threshold and up to approximately $28 \mathrm{~dB}$, the $2 \times 2$-MIMO outperforms the SISO system. For SNR above $27 \mathrm{~dB}$, the $3 \times 3$-MIMO becomes the best performance solution. It is only above $40 \mathrm{~dB}$ SNR that the full beam system surpasses the latter system. 


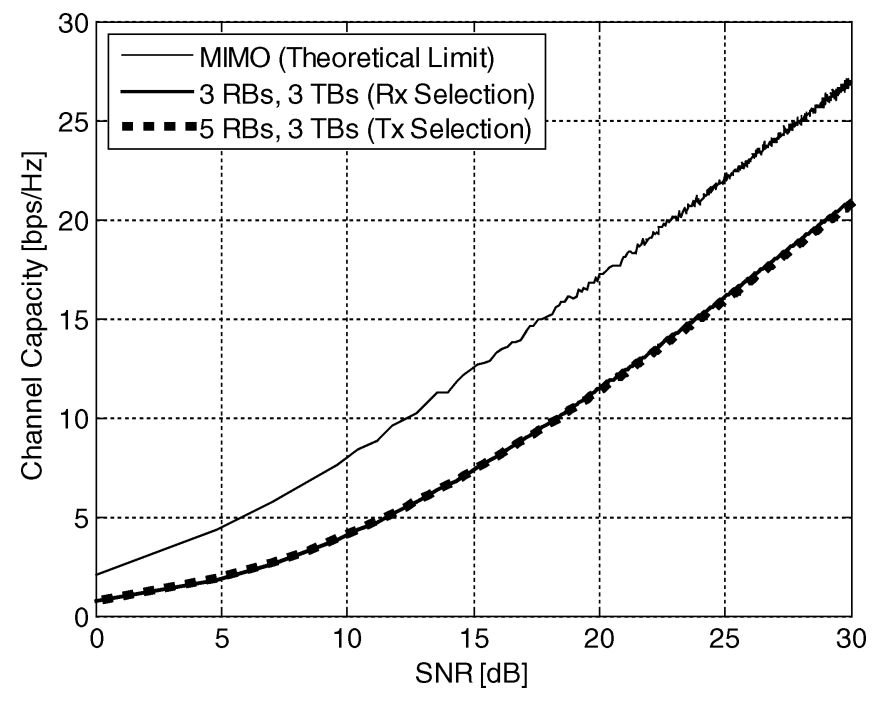

Fig. 8. Performance comparison for transmitter, receiver and joint beam selections for setup 02 (NLOS).

In NLOS, the $3 \times 3-\mathrm{MIMO}$ system surpasses the $2 \times 2$-MIMO system only above $17 \mathrm{~dB}$ of SNR in contrast with the $27 \mathrm{~dB}$ required for the NLOS.

LOS and NLOS multibeam systems show difference performances. For the $3 \times 3-\mathrm{MIMO}$, the capacity in NLOS case is $7 \mathrm{bps} / \mathrm{Hz}$ higher than that of LOS, at $20 \mathrm{~dB}$ SNR and the difference rises to $11 \mathrm{bps} / \mathrm{Hz}$ at $30 \mathrm{~dB}$ SNR.

\section{J. Discussion}

In order to make a quantitative analysis of the obtained results, the theoretical upper bound for MIMO systems needs to be introduced. The ergodic upper bound capacity for random channel (NLOS) can be expressed as [7]

$$
C=E_{H}\left\{N_{T} \log _{2}\left(1+\frac{\mathrm{SNR}}{N_{T}} \chi_{2 N_{R}}^{2}\right)\right\}
$$

where $E_{H}$ denotes the expectation over all channel realizations and $\chi_{2 N_{R}}^{2}$ is a chi-squared random variable with $2 N_{R}$ degrees of freedom. Only the results from setup 02 were compared with the theoretical bound since the Rayleigh-fading assumption does not hold for LOS (setup 01).

The best performance results from transmitter, receiver and joint selection are compared in Fig. 8. The number of TBs was limited to three for the sake of the comparison. The $3 \times 3$-MIMO system obtained after joint and receiver beam selection is equivalent. In other words, both optimization methods lead to the same combination of beams. However, the $5 \times 3$-MIMO system has the same performance as the $3 \times 3$-MIMO and therefore the latter is the optimum multibeam system. The optimum system has a capacity of $10 \mathrm{bps} / \mathrm{Hz}$ below the theoretical limit for SNR above $15 \mathrm{~dB}$.

\section{CONCLUSION}

The performance of multibeam MIMO systems in indoor wireless channels was studied based on empirical indoor measurements. Typical LOS and NLOS scenarios were characterized by using a virtual multibeam MIMO test bed consisting of mechanically steered horn antennas. It was found that the best multibeam system is the one that exploits the space-time diversity better. The optimum MIMO system is the one that uses the maximum number of channels, minimizing the interference and maximizing the SNR for each received beam ([7] and [8]). Using the system capacity as performance indicator, an exhaustive search algorithm was implemented to find the optimum combination of TB, RB, or both.

In the LOS case, the receiver beam selection has no considerable impact in the system capacity when all TBs are used simultaneously. The maximum achievable capacity performing receiver beam selection can be reached by using the beam angle that is the closest one to the dominant DOA. The same conclusion can be made for transmitter beam selection when all RBs are used. The system capacity can be improved for SNR below $17 \mathrm{~dB}$ by selecting the single $\mathrm{TB}$ angle that maximizes the received power, in our case $90^{\circ}$. It can be concluded that the capacity increases with the number of TBs and with the SNR in LOS. In terms of joint beam selection, the SISO system proved to be optimal in LOS for SNR below $18 \mathrm{~dB}$. The $2 \times 2$-MIMO system performed better for SNRs between 18 and $28 \mathrm{~dB}$. Finally, the $3 \times 3$-MIMO surpasses the full beam system for SNR up to $40 \mathrm{~dB}$.

The results for the NLOS case led to quite different conclusions. By means of receiver beam selection, more than $7 \mathrm{bps} / \mathrm{Hz}$ gain was achieved by using $2 \mathrm{RB}$ s instead of using a single RB at the working SNR of $27.8 \mathrm{~dB}$. By using 3 RBs instead of $2 \mathrm{RBs}$, a $2.5 \mathrm{bps} / \mathrm{Hz}$ improvement in capacity was observed. Therefore, it can be concluded that for receiver beam selection in NLOS, the improvement rate decreased as the number of RBs increases. After implementing transmitter beam selection, it was observed that a single TB was optimum for very low SNR. For SNR below $15 \mathrm{~dB}, 2$ TBs showed better performance. However, for higher SNR levels, the full beam system exhibits the best results in terms of capacity.

It is important to point out the difference between LOS and NLOS system performance. In LOS case, the maximum capacity obtained by any of the beam optimization methods never exceeded $7 \mathrm{bps} / \mathrm{Hz}$. However, for the same SNR, the NLOS capacity was about twice of that for LOS case, for any case of beam selection. This is a clear consequence of the amount of cross-correlation between space-time channels in LOS.

\section{ACKNOWLEDGMENT}

The authors would like to express their thanks to Ms. A. Emami and Mr. B. Tabachnick for their help with the measurements.

\section{REFERENCES}

[1] G. J. Foschini and M. J. Gans, "On the limits of wireless communications in a fading environment when using multiple antennas," Wireless Per. Commun., vol. 6, pp. 311-335, Mar. 1998.

[2] A. A. M. Saleh and R. A. Valenzuela, "A statistical model for indoor multipath propagation,” IEEE J. Select. Areas Commun., vol. 5, no. 2, pp. 128-138, Feb. 1987.

[3] M. Gharavi, Alkhansari, and A. Greshman, "Fast antenna selection in MIMO systems," IEEE Trans. Signal Process., vol. 52, no. 2, pp. 339-347, Feb. 2004

[4] Gorokhov, "Receive antenna selection for MIMO spatial multiplexing: Theory and algorithms," IEEE Trans. Signal Process., vol. 51, no. 11, pp. 2796-2807, Nov. 2000. 
[5] C. Balanis, Antenna Theory, Analysis and Design, 2nd ed. New York: Wiley, 1996.

[6] Q. H. Spencer, B. D. Jeffs, M. A. Jensen, and A. L. Swindlehurst, "Modeling the statistical time and angle of arrival characteristics of an indoor multipath channel," IEEE J. Select. Areas Commun., vol. 18, no. 3, Mar. 2000.

[7] B. Holter, "On the capacity of the MIMO Channel - A tutorial Introduction," Norwegian University of Science and Technology, Dept. Telecomm. [Online]. Available: bholter@tele.ntnu.no

[8] S. Sanayei and A. Nosratinia, "Antenna selection in MIMO systems," IEEE Commun. Mag., vol. 42, no. 10, pp. 68-73, Oct. 2004.

[9] T. Rappaport, Wireless Communications: Principles and Practice. Englewood Cliffs, NJ: Prentice Hall, 2001, ch. 5.

[10] A. V. Oppenheim and R. W. Shaffer, Discrete-Time Signal Processing, 2nd ed. Englewood Cliffs, NJ: Prentice Hall, 1999, Signal Processing Series, pp. 468-469.

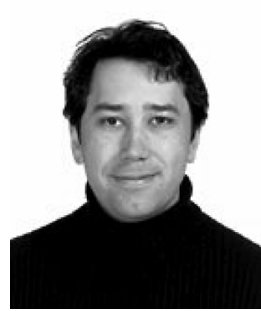

Luis E. Gurrieri received the B.E. degree in electronic engineering specializing in telecommunications from the University of Buenos Aires, Argentina, in 1998 and the M.Sc. degree from the University of Manitoba, Winnipeg, MB, Canada, in 2006. He is currently working towards the Ph.D. degree at Queen's University, Kingston, ON, Canada

From 1998 to 2005, he worked on a number of engineering and R\&D projects related to telecommunication systems. Since 2005, he has been working at the Communications Research Centre (CRC) in Ottawa, Canada, where he is currently a Research Engineer with the Terrestrial Wireless System group. His research interests include advanced wireless communication systems, radio propagation, channel modeling, and cognitive ratio.

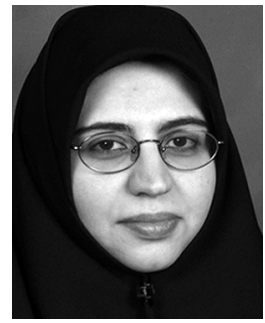

Sima Noghanian (M'03-SM'05) received the B.Sc. degree in electrical engineering from Sharif University of Technology, Tehran, Iran, in 1992 and the M.Sc. and Ph.D. degrees, both in electrical engineering, from the University of Manitoba, Winnipeg, MB, Canada, in 1996 and 2001, respectively.

In 2001, she worked for YottaYotta Corp., Edmonton, Canada, and in 2002 she received a postdoctoral fellowship from the Natural Sciences and Engineering Research Council of Canada (NSERC), which she took at the University of Waterloo. From 2002 to 2003, she was an Assistant Professor in the Electrical Engineering Department at Sharif University of Technology. Since 2003, she has been an Assistant Professor in the Electrical and Computer Engineering Department at the University of Manitoba. Her research interest includes antenna design and modeling with applications in wireless communication, wireless channel modeling, ultrawideband antennas and microwave imaging, and application of optimization in antenna design and imaging.

Dr. Noghanian served as the IEEE Winnipeg Waves (joint chapter of Antenna and Propagation/Microwave Theory and Techniques/Vehicular Technology societies) chair in 2004 and 2005. 